\title{
Multi-species biofilm of Candida albicans and non-Candida albicans Candida species on acrylic substrate
}

\author{
Apurva K PATHAK ${ }^{1}$, Sanjay SHARMA ${ }^{2}$, Pallavi SHRIVASTVA ${ }^{3}$ \\ 1- MSc. PhD, Sr. Lecturer, Department of Microbiology, Modern Dental College \& Research Centre, Indore (M.P.), India. \\ 2- BDS, MDS, Department of Prosthodontics with Crown, Bridge \& Implantology, Modern Dental College \& Research Centre, Indore (M.P.), India. \\ 3- BDS, MDS, Department of Oral Medicine, Diagnosis \& Radiology, Modern Dental College \& Research Centre, Indore (M.P.), India.
}

Corresponding address: Dr. Apurva K Pathak - Department of Microbiology, Modern Dental College \& Research Centre - Gandhinagar - Indore (M.P.) - India - 453112 - Phone: +91 7312882695 - Ext. 341 - Fax: +91 7312882697 - e-mail: pathak.apurva@gmail.com

Received: November 17, 2009 - Modification: February 16, 2010 - Accepted: March 19, 2010

\section{ABSTRACT}

\begin{abstract}
$\mathrm{O}$ bjective: In polymicrobial biofilms bacteria extensively interact with Candida species, but the interaction among the different species of the Candida is yet to be completely evaluated. In the present study, the difference in biofilm formation ability of clinical isolates of four species of Candida in both single-species and multi-species combinations on the surface of dental acrylic resin strips was evaluated. Material and Methods: The species of Candida, isolated from multiple species oral candidiasis of the neutropenic patients, were used for the experiment. Organisms were cultured on Sabouraud dextrose broth with $8 \%$ glucose (SDB). Biofilm production on the acrylic resins strips was determined by crystal violet assay. Student's t-test and ANOVA were used to compare in vitro biofilm formation for the individual species of Candida and its different multi-species combinations. Results: In the present study, differences between the mean values of the biofilm-forming ability of individual species (C. glabrata $>$ C. krusei>C. tropicalis $>$ C. albicans) and in its multispecies' combinations (the highest for $C$. albicans with $C$. glabrata and the lowest for all the four species combination) were reported. Conclusions: The findings of this study showed that biofilm-forming ability was found greater for non-Candida albicans Candida species (NCAC) than for $C$. albicans species with intra-species variation. Presence of $C$. albicans in multi-species biofilms increased, whereas; $C$. tropicalis decreased the biofilm production with all other NCAC species.
\end{abstract}

Key words: Oral candidiasis. Biofilms. Crystal violet. Assay.

\section{INTRODUCTION}

Candida is the most frequently isolated fungal pathogen in humans causing a variety of afflictions ranging from superficial mucosal infections to systemic mycoses. Oral fungal infections develop frequently in immunocompromised patients, particularly in patients with prolonged, severe neutropenic episodes ${ }^{2}$. In vivo studies indicate that microbial contamination of denture acrylic resin occurs quite rapidly and implanted devices like denture prostheses provide refuge to candidal organisms as either single-species or multi-species biofilms ${ }^{5,13}$. Consequently, the immunocompromised denture wearers are more prone to the fungal infections.

One of the major factors contributing to the virulence of Candida is its ability in acclimatize to a variety of different habitats for growth and formation of surface-attached microbial communities known as "biofilms". Biofilms are defined as microbial communities encased in a matrix of extracellular polymeric substance (EPS), which display phenotypic features that differ from their planktonic or free-floating counterparts ${ }^{7,16,19}$.

In vitro studies indicate that microbial contamination of denture acrylic resin occurs quite rapidly and the yeast cells adhere strongly to denture materials $s^{4,13,18}$. As bacteria and non-Candida albicans Candida species are often found with 
Candida albicans in polymicrobial biofilms in vivo, it is likely that extensive interspecies interactions take place in these adherent populations $\mathbf{s}^{16,17,19}$.

A large number of in vitro model systems have been used to investigate characteristics of singlespecies and mixed-species biofilms consisting of $C$. albicans and bacteria ${ }^{1,3,10,11,12,17}$. Some studies have also been done on the interaction between different species of Candida on in vitro dual-species biofilm formation ${ }^{20,25}$. The study on the interaction between different species of Candida on in vitro multi-species biofilm formation is still scarce ${ }^{27}$.

In the present study, the differences in biofilmforming ability of clinical isolates of four species of Candida in single-species and multi-species combination on the surface of dental acrylic resin strips, commonly used for dental appliances, was evaluated.

\section{MATERIAL AND METHODS}

\section{Organisms}

The test organisms included 24 isolates of 4 different species of Candida i.e. C. albicans, C. kruzi, C. glabrata and C. tropicalis (six isolates of each), isolated from the oral lesions of multiple species ( $\geq 2$ Candida species) Oropharyngeal Candidiasis (OPC) of the neutropenic patients (absolute neutrophil count $<1.5 \times 10^{9}$ cells $\left./ L\right)^{28}$. Pure culturing and identification was done at the Department of Microbiology, Modern Dental College and Research Centre. The identification of Candida species was conducted by culture characteristics on HiChrome Candida agar medium (HiMedia, Mumbai, India), assessing germ tube, chlamydospore formation and sugar assimilation patterns $\mathbf{s}^{6,22}$.
Simulated acrylic resins plates fabrication

Thirty-six square acrylic resins (polymethylmethacrylate) strips of $10 \times 10 \times 3 \mathrm{~mm}$ were fabricated. The acrylic resins strips were prepared as described by Samaranayake and MacFarlane ${ }^{21}$ (1980) with some modifications. Wax patterns were invested in denture flasks, boiled out, packed with the denture base resins, and heat polymerized according to manufacturer instructions at a temperature $73^{\circ} \mathrm{C}$ for $6 \mathrm{~h}$. Strips were removed from flask after bench cooling. One surface of strips was polished on buff wheel with pumice slurry. Other surface was left untouched to simulate intaglio surface. The resultant acrylic resins strips were immersed in distilled water for 1 week to leach excess monomer. Following this strips were disinfected by dipping in $70 \%$ alcohol for $1 \mathrm{~min}$, washed with sterile distilled water, dried and used for the experiment after checking their sterility.

\section{Determination of biofilm production}

Sabouraud dextrose broth (SDB) prepared from powdered Sabouraud broth (HiMedia, Mumbai, India) supplemented with $60 \mathrm{~g}$ of glucose per liter (final glucose concentration, $80 \mathrm{~g} /$ liter or $8 \%$ ) (Qualigens, Navi Mumbai, India), was according to manufacturer's instructions. Fresh pure cultures of testing organism were prepared on SDA medium by subculturing clinical isolate. A loop full of organisms from each SDA plate was inoculated into modified Sabouraud dextrose broth ( $8 \%$ of glucose concentration) for $24 \mathrm{~h}$ at $35 \pm 2^{\circ} \mathrm{C}$. The turbidity of each suspension was adjusted to the equivalent of $1 \times 10^{7} \mathrm{CFU} / \mathrm{mL}$ with SDB as determined by comparative plate counts.

Next, $1 \mathrm{~mL}$ of suspension of isolated species and testing combination of different species was

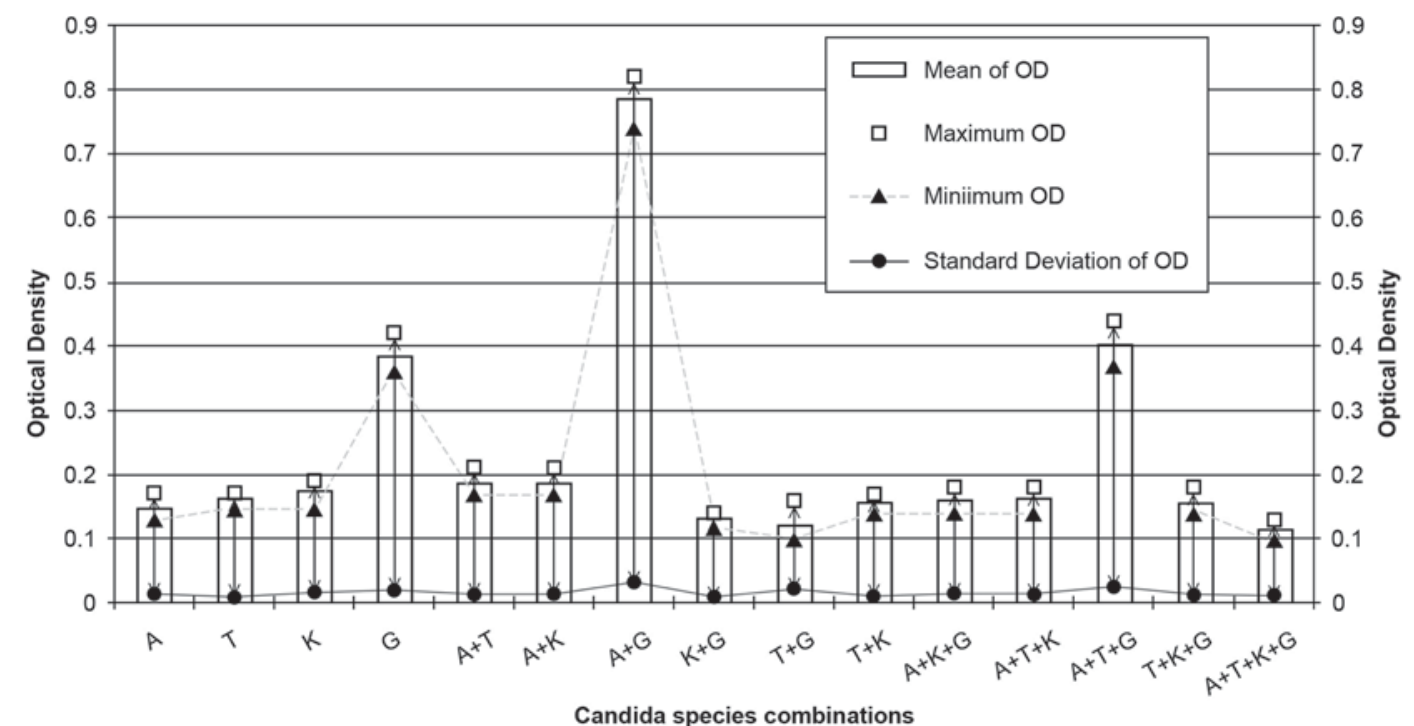

\# OD., Optical Density; A., C. albicans; T., C. tropicalis; K., C. krusei; G., C. glabrata; +, Combination of tested species

Figure 1- Optical Density of de-staining solution \# 
prepared by mixing equal volume of tested species, inoculated into a test tube with a screw cap (HiMedia, Mumbai, India) containing $9 \mathrm{~mL}$ of SDB, to make the final turbidity of each suspension to $1 \times 10^{6} \mathrm{CFU} / \mathrm{mL}$. Strips were placed in SDB, and then incubated at $35 \pm 2{ }^{\circ} \mathrm{C}$ for $24 \mathrm{~h}$ without agitation. After $24 \mathrm{~h}$ of incubation, the culture broth in the tube was aspirated gently, and then acrylic strips were taken out for further investigation.

The acrylic resins strips, on which biofilms developed, were washed once with distilled water, and then incubated in a crystal violet (HiMedia, Mumbai, India) staining solution $(0.1 \%$ in distilled water) for $15 \mathrm{~min}$. These were then washed three times with distilled water. The stain was then dissolved in de-staining solution ( $95 \%$ ethanol) and absorbance in terms of optical density (OD) was measured at $570 \mathrm{~nm}$ as previously described ${ }^{15}$. Untreated acrylic strips were used as a control for the amount of the crystal violet stain in the destaining solution. The absorbance values of controls were subtracted from the test values to minimize

Table 1- Paired samples t-test of Candida species combinations $\$$

\begin{tabular}{|c|c|c|c|c|}
\hline \multicolumn{2}{|c|}{ Pairs } & \multirow{2}{*}{$\frac{\text { t-test }}{-5,452}$} & \multirow{2}{*}{$\frac{\text { Significance level (2-tailed) }}{0,003}$} & \multirow{2}{*}{$\frac{\text { Decision }(\alpha=0.05)}{\text { Significant }}$} \\
\hline Pair 1 & $A-A+T$ & & & \\
\hline Pair 2 & $A-A+K$ & $-6,928$ & 0,001 & Significant \\
\hline Pair 3 & $A-A+G$ & $-65,11$ & 0 & Significant \\
\hline Pair 4 & $A-A+K+G$ & $-2,907$ & 0,034 & Significant \\
\hline Pair 5 & $A-A+T+K$ & $-2,697$ & 0,043 & Significant \\
\hline Pair 6 & $A-A+T+G$ & $-31,55$ & 0 & Significant \\
\hline Pair 7 & $A-A+T+K+G$ & 5,423 & 0,003 & Significant \\
\hline Pair 8 & $\mathrm{~T}-\mathrm{A}+\mathrm{T}$ & $-4,443$ & 0,007 & Significant \\
\hline Pair 9 & $T-T+G$ & 5,477 & 0,003 & Significant \\
\hline Pair 10 & $\mathrm{~T}-\mathrm{T}+\mathrm{K}$ & 1 & 0,363 & Non-significant \\
\hline Pair 11 & $T-A+T+K$ & 0 & 1 & Non-significant \\
\hline Pair 12 & $T-A+T+G$ & $-29,39$ & 0 & Significant \\
\hline Pair 13 & $T-T+K+G$ & 0,889 & 0,415 & Non-significant \\
\hline Pair 14 & $T-A+T+K+G$ & 8,367 & 0 & Significant \\
\hline Pair 15 & $K-A+K$ & $-3,503$ & 0,017 & Significant \\
\hline Pair 16 & $K-K+G$ & 8,73 & 0 & Significant \\
\hline Pair 17 & $K-T+K$ & 4,392 & 0,007 & Significant \\
\hline Pair 18 & $K-A+K+G$ & 2,169 & 0,082 & Non-significant \\
\hline Pair 19 & $K-A+T+K$ & 7 & 0,001 & Significant \\
\hline Pair 20 & $\mathrm{~K}-\mathrm{T}+\mathrm{K}+\mathrm{G}$ & 5 & 0,004 & Significant \\
\hline Pair 21 & $\mathrm{~K}-\mathrm{A}+\mathrm{T}+\mathrm{K}+\mathrm{G}$ & 8,919 & 0 & Significant \\
\hline Pair 22 & $G-A+G$ & $-32,85$ & 0 & Significant \\
\hline Pair 23 & $G-K+G$ & 27,568 & 0 & Significant \\
\hline Pair 24 & $G-T+G$ & 21,422 & 0 & Significant \\
\hline Pair 25 & $G-A+K+G$ & 20,684 & 0 & Significant \\
\hline Pair 26 & $G-A+T+G$ & $-1,025$ & 0,352 & Non-significant \\
\hline Pair 27 & $G-T+K+G$ & 37,997 & 0 & Significant \\
\hline Pair 28 & $G-A+T+K+G$ & 60,374 & 0 & Significant \\
\hline Pair 29 & $A+T-(A, T)$ & 5,27 & 0,003 & Significant \\
\hline Pair 30 & $A+K-(A, K)$ & 5,966 & 0,002 & Significant \\
\hline Pair 31 & $A+G-(A, G)$ & 50,747 & 0 & Significant \\
\hline Pair 32 & $K+G-(K, G)$ & $-23,95$ & 0 & Significant \\
\hline Pair 33 & $T+G-(T, G)$ & $-16,84$ & 0 & Significant \\
\hline Pair 34 & $\mathrm{~T}+\mathrm{K}-(\mathrm{T}, \mathrm{K})$ & $-3,841$ & 0,012 & Significant \\
\hline Pair 35 & $A+K+G-(A, K, G)$ & $-11,87$ & 0 & Significant \\
\hline Pair 36 & $\mathrm{~A}+\mathrm{T}+\mathrm{K}-(\mathrm{A}, \mathrm{T}, \mathrm{K})$ & 0,183 & 0,862 & Non-significant \\
\hline Pair 37 & $A+T+G-(A, T, G)$ & 16,021 & 0 & Significant \\
\hline Pair 38 & $\mathrm{~T}+\mathrm{K}+\mathrm{G}-(\mathrm{T}, \mathrm{K}, \mathrm{G})$ & $-30,62$ & 0 & Significant \\
\hline Pair 39 & $\begin{array}{c}A+T+K+G- \\
(A, T, K, G)\end{array}$ & $-25,85$ & 0 & Significant \\
\hline
\end{tabular}

${ }^{\$}$ C. albicans; T., C. tropicalis; K., C. krusei; G., C. glabrata; +, Combination of tested species; (,),Average of the biofilmforming ability of tested species independently. 
background interference.

\section{Statistical analysis}

The ODs of the amount of the crystal violet in the de-staining solution, measured for different Candida species, were compared by the paired Student's t-test by using the SPSS Win 12.0 program (SPSS Inc, Chicago, IL, USA). Differences between the isolated species and its multi-species combinations were considered to be significant for $P$ of 0.05 . The null hypothesis ( $\mathrm{HO}$ ) rejected in favor of the alternative hypothesis $(\mathrm{H} 1)$ at significance level a (0.05) if; $T>t_{n-1}, \alpha / 2$ (value of the Student table with $n-1$ degrees of freedom). The null hypothesis is $\mathrm{HO}: \delta=0$ (there is no difference in biofilm formation ability among the tested species) and the alternative hypothesis is (was) $\mathrm{H} 1: \delta=0$ (there is a difference in biofilm formation ability among the tested species).

\section{RESULTS}

Twenty-four Candida species isolated from clinical samples and were used for this study. Amongst the 24 isolates, 6 each of $C$. albicans, $C$. glabrata, C. krusei, and C. tropicalis were used to test the biofilm formation ability both in singlespecies and in multi-species combination. All the isolates produce moderate to high degree of biofilms on the surface of acrylic material (Figure 1 ). The biofilm-forming ability of C. glabrata were reported to be the highest on acrylic substances (OD - 0.3833 \pm 0.02066 ), whereas; of C. albicans to be the lowest (OD $-0.1467 \pm 0.01366)$. In case of multiple species conditions, it was observed that the $C$. albicans had a positive impact on biofilms formation on acrylic substances as the highest degree of slim production occurred when C. albicans were inoculated with C. glabrata

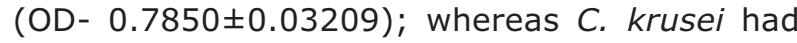
a negative impact in combination. Under multiple species condition when all the four species were in inoculums, the biofilms forming activity were severely hampered (OD $-0.1133 \pm 0.01033$ ).

To test the hypothesis of no difference or no relationship between biofilm-forming ability of isolated species with that of in-combination of other species, paired t-test was performed (Table 1). For all the four species, 7 different multi-species combinations were prepared, which made a total of 39 pairs. Eighty-five percent of the pairs rejected null hypothesis in favor of alternate hypothesis. Only $15 \%$ of pairs showed no difference between the mean values of the biofilm-forming ability of single-species and in its multi-species combination.

\section{DISCUSSION}

In patients with advanced immunodeficiency, mucosal infections can lead to severe oral and esophageal candidiasis, resulting in disseminated candidiasis and sometimes early death. One of the most important virulence factors of Candida species is its ability to form biofilms, which has an important clinical consequence, as it confers resistance to antifungal therapy and capacity for yeast cells within the biofilms to withstand host immune defenses $3,5,6,12,19$. Changes in the oral environment effected by tooth loss or denture wearing can cause changes in oral microflora. The carriage rates of single and multiple Candida species were reported to be significantly higher in denture wearers ${ }^{11}$. In the light of above fact Candida, isolated from multiple species candidimia lesion, were evaluated for their biofilms formation ability on acrylic surface in vitro, in single-species and multi-species combination.

Candida biofilms formation has been described on polymethylmethacrylate strips which occur essentially in three overlapping phases: early (0-11 h), intermediate (12-30 h), and maturation (38$72 \mathrm{~h}$ ) phases. The early stage is characterized by adherence and development of blastospores into distinct microcolonies. By 18 to $24 \mathrm{~h}$, the Candida biofilm community can be seen as a bilayered structure comprising a mixture of yeasts, germ tubes, and young hyphae; this intermediate phase is distinguished by the production of extracellular polymeric substance (EPS). During maturation, the biofilms becomes a thick EPS layer in which a dense network of yeasts, pseudohyphae, and hyphae are embedded ${ }^{4,18}$.

The biofilm-forming ability of clinical isolates of C. albicans, C. krusei, C. tropicalis and C. glabrata recovered from multiple species candidimia lesions, was evaluated by measuring absorbance of the de-staining solution containing crystal violet dye (crystal violet assay). The present showed $C$. glabrata forming thickest slim layer on acrylic resins strip followed by $C$. krusei, C. tropicalis and C. albicans, contrasting with the findings of previous researchers ${ }^{9,24}$. However Silva, et al. ${ }^{23}$ (2009) reported that C. glabrata biofilms matrix was high in both protein and carbohydrates, which that probably enabled it to adsorbed more amount of crystal violet contents.

Shin, et al.22 (2002) observed that biofilm formation was most frequent for isolates of $C$. tropicalis (80\%), followed by C. parapsilosis (73\%), C. glabrata $(28 \%)$, and C. albicans $(8 \%)$. This finding contrasts with the present adhesion studies, probably due to the sources of isolates. Different strains of the same Candida species were found to be different in their ability to form biofilms was 
also reported in this study, indicating "strong" and "weak" biofilms-forming strains might exist within each Candida species ${ }^{26}$. Shin, et al. ${ }^{22}$ (2002) also reported that biofilms-forming ability was greater for NCAC than for albicans species using similar protocol.

Presence of one species of microorganism on a surface can promote the adhesion of another ${ }^{14}$. Thein, et al. ${ }^{25}$ (2007) reported competitive interaction in a dual-species biofilms of $C$. albicans and C. Krusei; whereas Pereira-Cenci, et al.20 (2008) did not report competitive interaction in a dual-species biofilms of $C$. albicans and $C$. glabrata. In the present study, the highest degree of slim production was seen with the combination of $C$. albicans and $C$. glabrata, followed by the combination of $C$. albicans, $C$. tropicalis and $C$. glabrata, and the lowest slim production was seen with the multi-species biofilms of all the four species of Candida. Presence of C. albicans in multi-species biofilms increased the production of slim when inoculated with all other NCAC species; whereas C. tropicalis retarded the production of slim under multi-species condition except with $C$. albicans. In this way, C. albicans might be able to successfully provide a substratum to the NCAC species on the acrylic prosthesis.

One of the most significant features of microbial biofilms is its resistance to a variety of antimicrobial agents. Studies have demonstrated drug resistance when Candida biofilms are even grown on surfaces like denture acrylic. The possible mechanisms of biofilm resistance to antimicrobial agents are: restricted penetration of drugs through the biofilms matrix; phenotypic switching, surface-induced expression of resistance genes and a small number of "persistent" cells 8 . Presence of two or more species in a biofilm could aggregate these factors. Synergistic effects of these factors can pose major problems to the clinicians.

\section{CONCLUSION}

Based on the results of this study, biofilmforming ability was found greater for NCAC than for albicans species, isolated from multi-species oral candidiasis of the neutropenic patients. Presence of C. albicans in multi-species biofilms increased the slim production with all other NCAC species, whereas $C$. tropicalis impeded the production of slim under multi-species condition except for $C$. albicans.

\section{ACKNOWLEDGEMENTS}

Authors are greatly thankful to Dr. P.V. Wanjari, Honorable Dean, Modern Dental College and Research Centre, Indore, for providing academic and materialistic supports, without which it was hard to perform this work. We also acknowledge the helpful comments and suggestions of the reviewers of JAOS.

\section{REFERENCES}

1- Adam B, Baillie GS, Douglas LJ. Mixed species biofilms of Candida albicans and Staphylococcus epidermidis. J Med Microbiol. 2002; 51:344-9.

2- Alberth M, Majoros L, Kovalecz G, Borbás E, Szegedi I, Márton IJ, et al. Significance of oral Candida infections in children with cancer. Path Onco Res. 2006;12:237-41

3- Baillie GS, Douglas LJ. Candida biofilms and their susceptibility to antifungal agents. Methods Enzymol. 1999;310:644-56.

4- Chandra J, Kuhn DM, Mukherjee PK, Hoyer LL, McCormick T, Ghannoum MA. Biofilm formation by the fungal pathogen Candida albicans: development, architecture, and drug resistance. ] Bacteriol. 2001;183:5385-94.

5- Coogan MM, Fidel PL Jr, Komesu MC, Maeda N , Samaranayake LP. Candida and mycotic Infections. Adv Dent Res. 2006;19:130-8. 6- Costa CR, Cohen AJ, Fernandes OF, Miranda KC, Passos XS, Souza LK, et al. Asymptomatic oral carriage of Candida species in HIV-infected patients in the highly active antiretroviral therapy era. Rev Inst Med Trop Sao Paulo. 2006;48:257-61.

7- Costerton JW. Overview of microbial biofilms. J Ind Microbiol. 1995; 15:137-40.

8- Douglas LJ. Medical importance of biofilms in Candida infections. Rev Iberoam Micol. 2002;19:139-43.

9- Hawser SP, Douglas LJ. Biofilm formation by Candida species on the surface of catheter materials in vitro. Infect Immun. 2002;62:915-21.

10- Henriques M, Azeredo J, Oliveira R. Candida albicans and Candida dubliniensis: comparison of biofilm formation in terms of biomass and activity. Br J Biomed Sci. 2006;63:5-11.

11- Jenkinson HF, Douglas LJ. Interactions between Candida species and bacteria in mixed infections. In: Brogden KA, Guthmiller JM, editors. Polymicrobial diseases. Washington DC: ASM Press; 2002. p. 357-73.

12- Jin YY, Yip HK, Samaranayake YH, Yau JY, Samaranayake LP. Biofilm-forming ability of Candida albicans is unlikely to contribute to high levels of oral yeast carriage in cases of human immunodeficiency virus infection. J Clin Microbiol. 2003;41:29617.

13- Kuhn DM, Chandra J, Mukherjee PK, Ghannoum MA. Comparison of biofilms formed by Candida albicans and Candida parapsilosis on bioprosthetic surfaces. Infect Immun. 2002;70:878-88.

14- Leung JW, Liu YL, Desta T, Libby E, Inciardi JF, Lam K. Is there a synergistic effect between mixed bacterial infection in biofilm formation on biliary stents? Gastrointest Endosc. 1998;48:250-7. 15- O'Toole GA, Kolter R. Flagellar and twitching motility are necessary for Pseudomonas aeruginosa biofilm development. Mol Microbiol. 1998;30:295-304.

16- Peeters E, Nelis HJ, Coenye T. Comparison of multiple methods for quantification of microbial biofilms grown in microtiter plates. J Microbiol Methods. 2008;72:157-65.

17- Penha SS, Birman EG, Silveira FR, Paula CR. Frequency and enzymatic activity (proteinase and phospholipase) of Candida albicans from edentulous patients, with and without denture stomatitis. Pesq Odontol Bras. 2000;14:119-122.

18- Pereira-Cenci T, Cury AA, Cenci MS, Rodrigues-Garcia RC. In vitro Candida colonization on acrylic resins and denture liners: influence of surface free energy, roughness, saliva, and adhering bacteria. Int J Prosthodont. 2007;20:308-10.

19- Pereira-Cenci T, Del Bel Cury AA, Crielaard W, Ten Cate JM. Development of Candida-associated denture stomatitis: new insights. J Appl Oral Sci. 2008;16:86-94. 
20- Pereira-Cenci T, Deng DM, Kraneveld EA, Manders EM, Del Bel Cury AA, Ten Cate JM, Crielaard W. The effect of Streptococcus mutans and Candida glabrata on Candida albicans biofilms formed on different surfaces. Arch Oral Biol. 2008;53:755-64.

21- Samaranayake LP, MacFarlane TW. An in vitro study of the adherence of Candida albicans to acrylic surfaces. Arch Oral Biol. 1980;25:603-9.

22- Shin JH, Kee SJ, Shin MG, Kim SH, Shin DH, Lee SK, et al. Biofilm production by isolates of Candida species recovered from nonneutropenic patients: comparison of bloodstream isolates with isolates from other sources. J Clin Microbiol. 2002;40:1244-8.

23- Silva S, Henriques M, Martins A, Oliveira R, Williams D, Azeredo J. Biofilms of non-Candida albicans Candida species: quantification, structure and matrix composition. Med Mycol. 2009;47:681-9.

24- ten Cate JM, Klis FM, Pereira-Cenci T, Crielaard W, de Groot PWJ. Molecular and cellular mechanisms that lead to Candida biofilm formation. J Dent Res. 2009;88:105-15.
25- Thein ZM, Samaranayake YH, Samaranayake LP. Characteristics of dual species Candida biofilms on denture acrylic surfaces. Arch Oral Biol. 2007;52:1200-8

26- Thein ZM, Samaranayake YH, Samaranayake LP. In vitro biofilm formation of Candida albicans and non-albicans Candida species under dynamic and anaerobic conditions. Arch Oral Biol. 2007;52:761-7.

27- Thein ZM, Seneviratne CJ, Samaranayake YH, Samaranayake LP. Community lifestyle of Candida in mixed biofilms: a mini review. Mycoses. 2009;52:467-75.

28- Watts RG. Neutropenia. In: Lee GR, Foerster J, Lukens J, Paraskevas F, Greer JP, Rodgers GM, et al, editors. Wintrobe's Clinical Hematology. Baltimore: Lippincott, Williams \& Wilkins; 1999. p. $1862-88$ 